PSICOLOGIA

IBEROAMERICANA
Psicología Iberoamericana ISSN: 1405-0943

revista.psicologia@ibero.mx

Universidad Iberoamericana, Ciudad de México México

\title{
Construcción de una escala de actividades de tiempo libre en padres de familia mexicanos
}

Valencia Camargo, María Fernanda; Díaz Loving, Rolando; Reyes Lagunes, Lucina Isabel; López Peñaloza, Judith; Cruz del Castillo, Cinthia

Construcción de una escala de actividades de tiempo libre en padres de familia mexicanos

Psicología Iberoamericana, vol. 26, núm. 1, 2018

Universidad Iberoamericana, Ciudad de México, México

Disponible en: http://www.redalyc.org/articulo.oa?id=133959553009 


\title{
Construcción de una escala de actividades de tiempo libre en padres de familia mexicanos
}

Development of a leisure time activities scale for mexican

parents

\author{
María Fernanda Valencia Camargo \\ mafernanda.valencia.c@gmail.com \\ Universidad Nacional Autónoma de México , México \\ Rolando Díaz Loving \\ Universidad Nacional Autónoma de México, México \\ Lucina Isabel Reyes Lagunes \\ Universidad Nacional Autónoma de México, México \\ Judith López Peñaloza \\ Universidad Michoacana de San Nicolás de Hidalgo, México \\ Cinthia Cruz del Castillo \\ Universidad Iberoamericana, México
}

Resumen: El objetivo del presente trabajo fue crear un instrumento para evaluar las actividades de tiempo libre en adultos. La influencia de las actividades de tiempo libre se ha estudiado en el desarrollo de conductas positivas y negativas que impactan al bienestar. La muestra constó de 587 padres de familia de la Ciudad de México, entre 25 y 65 años $(\mathrm{M}=44.30, \mathrm{DE}=9.34), 79.5 \%$ de la muestra estaban casados o cohabitaban. La escala constó de 121 actividades, los participantes indicaron la frecuencia de participación en una escala Likert de 1 (nunca) a 5 (muy a menudo). El análisis factorial exploratorio arrojó 13 factores (66 ítems), que explican $61.30 \%$ de la varianza, el coeficiente de consistencia interna fue 0.94 . La escala es una prueba válida y confiable de las actividades de tiempo libre, y puede ser utilizada satisfactoriamente en la investigación con muestras mexicanas.

Palabras clave: escala, tiempo productivo, tiempo libre, actividades, padres de familia mexicanos.

Abstract: The study aims to create an instrument, which evaluates past-time activities in adults. The influence of past-time activities has been studied in the development of positive behaviors and the avoidance of negative behaviors, which impact on wellbeing. The sample consisted of 587 parents of Mexico City, aged between 25 and 65-years-old $(\mathrm{M}=44.30, \mathrm{SD}=9.34), 79.5 \%$ reported being in a married or cohabiting relationship. The scale contained 121 past-time activities with respondents indicating the frequency of participation on a Likert scale from 1 (never) to 5 (very often). Exploratory factor analysis produced 13 factors (66 items), accounting for $61.30 \%$ of the variance. Internal consistency coefficient was 0.94 . The scale suggests a concise, valid and reliable test of past-time activities, and can be satisfactorily used with Mexican samples, which may be applied to future research.

Keywords: scale, productive time, leisure time, activities, Mexican parents.

\section{INTRODUCCIÓN}

El tiempo es un recurso fundamental de la vida (Damián, 2004) dividido en cuatro áreas: a) Tiempo psicobiológico, destinado a necesidades 
fisiológicas y psíquicas; b) Tiempo socioeconómico, destinado al trabajo; c) Tiempo sociocultural, dedicado a la vida en sociedad; y d) Tiempo de ocio/libre, dedicado al disfrute personal y colectivo (Munné \& Codina, 1996).

En la actualidad, la vida en sociedad (tiempo psicobiológico, socioeconómico y sociocultural) evidencia el anhelo de un tiempo personal, libre de compromisos y responsabilidades (Ángel, 2001). El tiempo libre es un espacio que ofrece la oportunidad de realizar actividades libremente elegidas que permiten el desarrollo de la creatividad, la autoestima, la autorrealización, el desarrollo personal y las relaciones sociales (Boltvinik, 2003).

La concepción de tiempo libre surge en la Revolución Industrial, cuando el fin máximo era la producción. Dicha situación generó en los trabajadores la toma de conciencia de su papel, esclavos del trabajo, donde el tiempo de descanso era apenas el necesario para recuperar fuerzas y regresar a la jornada laboral. La explotación de la fuerza de trabajo despertó diversas luchas sociales con el fin de disminuir el tiempo de trabajo y dar lugar al tiempo libre, un espacio de libertad, para usarlo a conveniencia y gusto personal (Ángel, 2001; Miquilena, 2011).

Desde mediados del siglo xx el uso del tiempo libre ha sido estudiado como un indicador importante del estudio de la situación social, el bienestar y la calidad de vida (Romo, 2003). El tiempo libre permite superar las necesidades e insatisfacciones de la vida diaria, debido a la libertad de elección presente es una fuente de creatividad e identidad (Munné \& Codina, 1996).

En el contexto familiar, la llegada de los hijos modifica la interacción del sistema. Las actividades de tiempo libre pierden la libertad de elección y motivación intrínseca. El tiempo libre es utilizado con el objetivo de mejorar el funcionamiento familiar y transmitir a los hijos valores y estilos de vida saludables (Shaw \& Dawson, 2001). El desarrollo teórico y empírico del tiempo libre ha prestado poca atención a la madre y al padre y ha centrado el estudio de los beneficios del tiempo libre al sistema como un todo (Shaw, 2008).

El antecedente en la evaluación del tiempo libre familiar es el Core and Balance Model of Family Leisure Functioning, desarrollado por Zabriskie y McCormick (2001) de las actividades de tiempo libre de instrumentos de evaluación del funcionamiento familiar. En dicho modelo, las actividades core son actividades dentro del hogar, caracterizadas por proporcionar estabilidad y regularidad; las actividades balance son aquellas que ofrecen al sistema novedad y cambio al proveer nuevas experiencias, son actividades fuera del hogar.

Investigaciones más recientes han evaluado el tiempo libre a nivel familiar (Claxton \& Perry-Jenkins, 2008; Lee \& Bhargava, 2004; Mattingly \& Sayer, 2006), los métodos de evaluación han incluido: a) Los cuestionarios, ya sea abiertos (si las personas reportan las actividades) o cerrados (existe una lista previamente diseñada); b) los diarios o bitácoras, en los cuales los participantes reportan las actividades que llevaron a cabo a lo largo del día; y c) diarios o bitácoras acompañados de medios 
electrónicos, en los cuales el dispositivo suena cada determinado tiempo, momentos en los cuales los participantes deben registrar la actividad que están llevando a cabo (Arteaga, Gayet, \& Alegría, 2016; Cruz-Sánchez, Moreno-Contreras, Pino-Ortega, \& Martínez-Santos, 2011; inegi, 2014, Gutiérrez, 2008; Munné \& Codina, 1996).

En México, la Encuesta Nacional sobre Uso del Tiempo (inegi, 2014) proporciona información de la distribución y uso del tiempo en hombres y mujeres de 12 años en adelante, sin embargo, dicha medición no proporciona una medida con características psicométricas adecuadas que permita evaluar la frecuencia de las actividades llevadas a cabo.

Adicionalmente, Mattingly y Sayer (2006) y Offer (2016) llevaron a cabo estudios para comparar la cantidad y calidad de tiempo libre entre padres y madres de familia, y reportan diferencias significativas. Es más probable que las madres tengan actividades no remuneradas en este espacio de tiempo o lo inviertan en el cuidado de los hijos. Mientras que los padres, a pesar del incremento en el involucramiento en la paternidad, pueden estar menos limitados en el uso del tiempo libre por las obligaciones de cuidado infantil.

En el presente estudio el tiempo libre es un indicador importante del bienestar; a nivel familiar la llegada de los hijos impacta en la distribución del mismo. En México es esencial contar con un instrumento con propiedades psicométricas adecuadas que permita evaluar el tiempo libre de manera objetiva y confiable para desarrollar investigación en torno al impacto de esta dimensión en madres y padres, y evaluar el efecto en múltiples facetas de la realidad social. La investigación se dividió en dos estudios cuyos objetivos fueron conocer las actividades de tiempo libre de madres y padres de familia y construir un instrumento con características psicométricas adecuadas para evaluar las actividades de tiempo libre de madres y padres de familia.

\section{ESTUDIO 1: MÉTODO}

\section{Participantes}

Se trabajó con una muestra no probabilística de 150 madres y padres de familia, 50 con hijos menores de 12 años, 50 con hijos de 13 a 18 años y 50 con hijos mayores de 19 años; con un rango de edad de 20 a 66 años $(\mathrm{M}=43.10, \mathrm{DE}=10.37)$. El 81.8\% de la muestra cohabitaba con su pareja. Respecto a la escolaridad, $34.9 \%$ de la muestra tenía educación básica concluida, $34.1 \%$ educación media superior y $31 \%$ educación superior.

\section{Instrumento}

Se aplicó a los participantes un cuestionario en el cual se les pedía registrar las actividades de tiempo libre llevadas a cabo recientemente y un apartado de socio-demográficos. 


\section{Procedimiento}

La aplicación se realizó de forma individual contactando a madres y padres en escuelas, sus hogares y espacios al aire libre. A cada uno de ellos se solicitó su participación voluntaria para contestar el cuestionario, garantizando la confidencialidad y el anonimato de los datos.

\section{Resultados}

Para obtener las categorías de las actividades de tiempo libre se llevó a cabo el análisis con teoría fundamentada (Hernández, Fernández, \& Baptista, 2010), a través del cual se transcribieron los datos (primera lectura), se revisaron (segunda lectura), se identificaron las unidades de análisis (ua) (actividades reportadas por los participantes), se codificaron las ua (categorías de actividades) y, finalmente, se codificaron las categorías (agrupar categorías y ejemplificar). Surgieron 17 categorías, que aparecen en la Tabla 1, así como algunos ejemplos de las actividades que conforman los reactivos de la Escala de Tiempo Libre a validar.

\section{ESTUDIO 2: MÉTODO}

\section{Participantes}

Se trabajó con una muestra no probabilística de 587 madres y padres de familia (52.6\% madres) con edades entre 25 y 65 años $(\mathrm{M}=44.30, \mathrm{DE}=$ 9.34), 79.5\% de la muestra estaban casados o cohabitaban con su pareja. Respecto a la escolaridad, $33.2 \%$ de la muestra tenía educación básica concluida, $33.9 \%$ educación media superior y $32.9 \%$ educación superior. Los participantes tenían en promedio de 2 a 3 hijos y eran residentes de la Ciudad de México y el Estado de México.

\section{Instrumento}

A partir del estudio exploratorio se diseñó un instrumento que contiene 121 actividades de tiempo libre, en el cual los participantes reportaron la frecuencia de participación en una escala tipo Likert de cinco opciones que van de $1=$ nunca a $5=$ muy frecuentemente.

\section{Procedimiento}

Se pidió la participación voluntaria a madres y padres de familia para responder el instrumento, cuyo objetivo era recabar información acerca de la interacción familiar, se garantizó la confidencialidad y el anonimato de los datos y se procedió con la aplicación. 
Para el análisis de los datos se llevó a cabo el procedimiento de validación de instrumentos propuesto por Reyes Lagunes y García y Barragán (2008).

Se llevó a cabo el análisis de frecuencias para cada uno de los reactivos, solicitando media, desviación estándar y sesgo a fin de verificar que no existan valores fuera de rango, la dirección y la distribución. Posteriormente, para evaluar la capacidad de discriminación de los reactivos, se generó una nueva variable equivalente al resultado de la suma de los reactivos de la escala, para obtener los valores del percentil 25 y 75 de esta variable, con lo cual se obtuvo una variable dicotómica basada en los cuartiles extremos. Dicha variable es la variable de agrupación para realizar la prueba t de Student. Paso seguido se analizó la direccionalidad a través de tablas cruzadas con los grupos extremos. Se obtuvo la Confiabilidad interna (Alfa de Cronbach), donde se revisaron los valores de correlación de cada reactivo con el total y el valor de modificación del estadístico de confiabilidad si se elimina el reactivo. Se llevó a cabo el análisis de correlación para determinar el tipo de rotación a utilizar, se obtuvo la composición factorial y, finalmente, el índice de confiablidad Alfa de Cronbach para el total de la escala y para cada factor que surgió. 


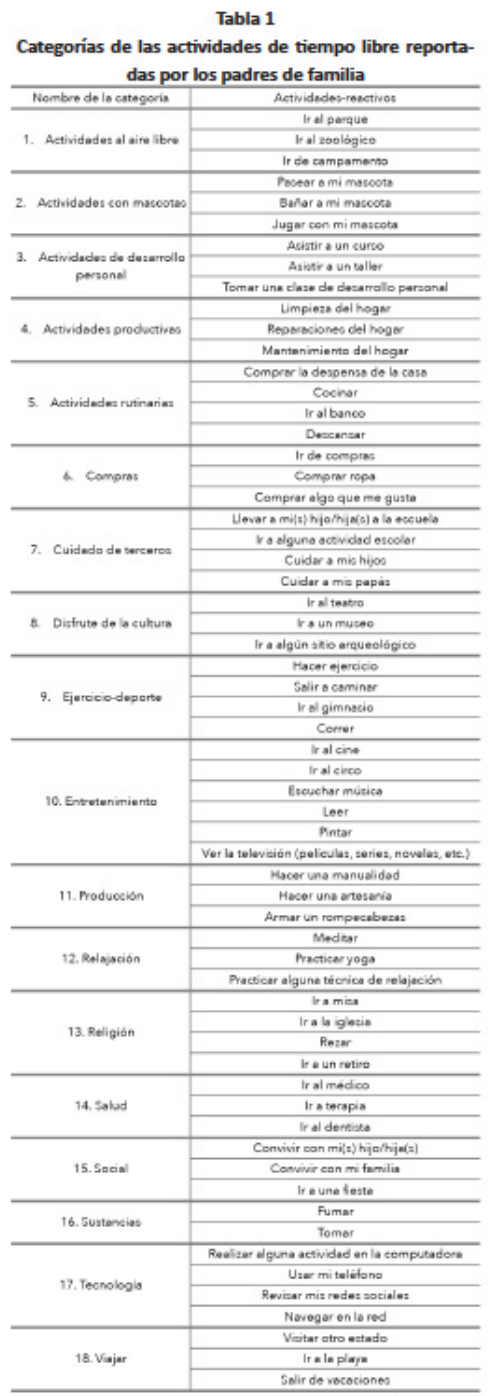

Tabla 1

Categorías de las actividades de tiempo libre reportadas por los padres de familia

\section{Resultados}

A partir de las pruebas de análisis de frecuencias, discriminación de reactivos para grupos extremos (prueba $t$ de Student), direccionalidad y correlación ítem-total mayor a 0.3 (Alfa de Cronbach), de los 121 reactivos originales, sólo 93 cumplieron los requisitos suficientes para ser considerados en el análisis final de los datos. Estos 93 reactivos fueron incluidos en un análisis factorial de componentes principales con rotación ortogonal (Varimax). Se eliminaron los reactivos con comunidades menores a 0.40 , se tomó la decisión de establecer 0.40 como el peso factorial mínimo, 1 como el valor propio mínimo para cada factor, y 3 el número mínimo de reactivos para cada factor.

Solo 66 de los 93 reactivos cumplieron con los requisitos ya establecidos y éstos se agruparon en 13 factores que explican $61.30 \%$ de la varianza total. La prueba de esfericidad de Bartlett fue significativa (16641.81, 
$\mathrm{gl}=2145, \mathrm{p}<.001)$ y la media de la adecuación del tamaño de muestra Kaiser Meyer-Olkin fue adecuado (.91). El análisis de consistencia interna mediante Alpha de Cronbach para la escala total arrojó un valor de .94.

En cuanto a los índices de consistencia interna obtenidos para cada factor éstos variaron de .65 (Actividades con música) a .88 (Escape de la rutina y Cuidado parental). La media más baja se obtuvo en el factor de Desarrollo personal y relajación (1.87) y la más alta en el factor de Actividades rutinarias (3.77) como se muestra en la Tabla 2. Los factores de la escala y los reactivos que componen los mismos aparecen en la Tabla 3.

Tabla 2

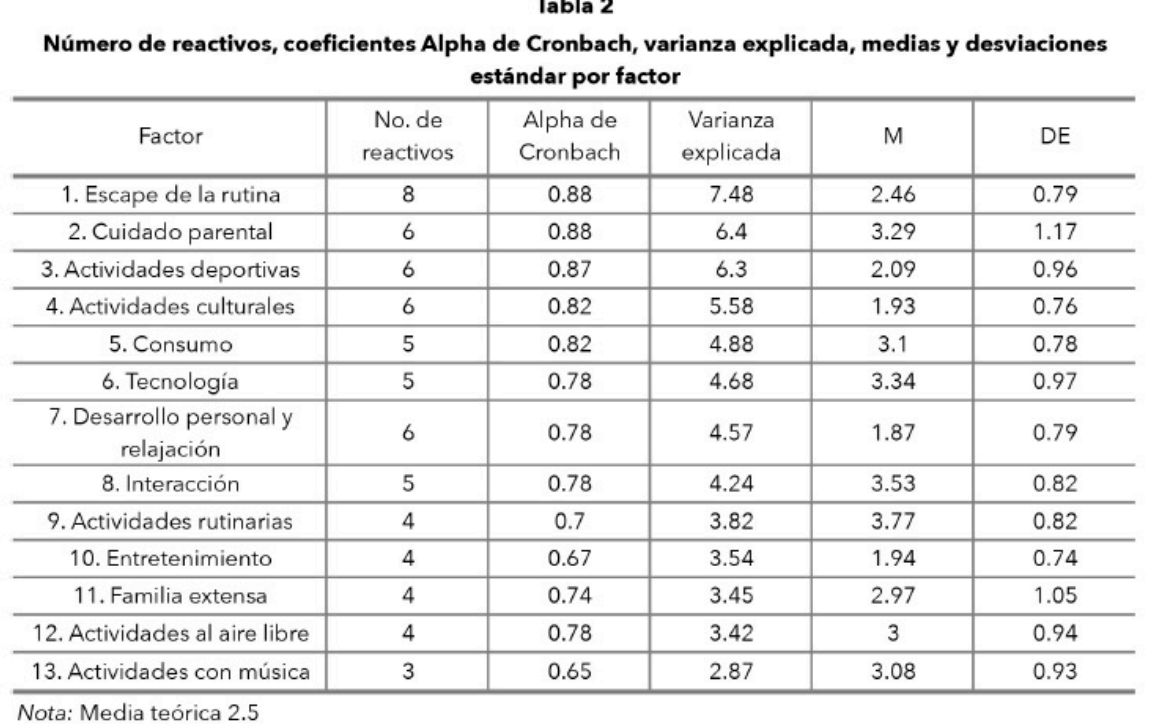

Tabla 2

Número de reactivos, coeficientes Alpha de Cronbach, varianza explicada, medias desviaciones estándar por factor

Adicionalmente se obtuvieron los coeficientes de Correlación de Pearson entre factores, dichas relaciones muestran asociaciones positivas entre todas las categorías de actividades, la correlación más alta (.60) aparece entre Desarrollo personal y relajación, y Actividades culturales, y la correlación más baja (.17) entre Entretenimiento y Tecnología (ver Tabla 4).

Con la intención de indagar si existen diferencias significativas en los factores de la escala entre el grupo de madres y padres, se realizaron pruebas $t$ de Student, los resultados arrojaron diferencias significativas en 7 de los 13 factores. Las mujeres obtuvieron medias más altas en cinco (Cuidado parental, Consumo, Actividades rutinarias, Familia extensa y Actividades con música) y los hombres obtuvieron medias más altas en dos de las categorías de actividades (Escape de la rutina y Actividades deportivas) (ver Tabla 5).

\section{DISCUSIÓN}

El tiempo libre es el espacio destinado a llevar a cabo actividades de disfrute personal (Munné \& Codina, 1996), aunque Shaw y Dawson 
(2001) y Shaw (2008) refieren al tiempo libre de madres y padres como un tiempo dedicado a la interacción familiar, permeando el tiempo libre personal. Dos de los factores de la escala, Cuidado parental (2) y Actividades rutinarias (9), aportan evidencia a favor de dichas investigaciones. Las actividades de tiempo libre de madres y padres involucran el cumplimiento de las funciones asignadas socialmente, el cuidado y buen funcionamiento del sistema familiar (García-Méndez, Rivera-Aragón, Díaz-Loving, \& Reyes Lagunes, 2015). Sin embargo, la mayoría de categorías refieren al tiempo libre a nivel personal.

En congruencia con la Encuesta Nacional sobre Uso del Tiempo (inegi, 2014), la escala muestra una amplia gama de actividades: personal (Desarrollo personal y relajación), social (Cuidado parental, Interacción y Familia extensa), rutina (Compras y Actividades rutinarias), pasatiempos (Entretenimiento y Actividades con música), Deportes, Consumo y Actividades culturales.
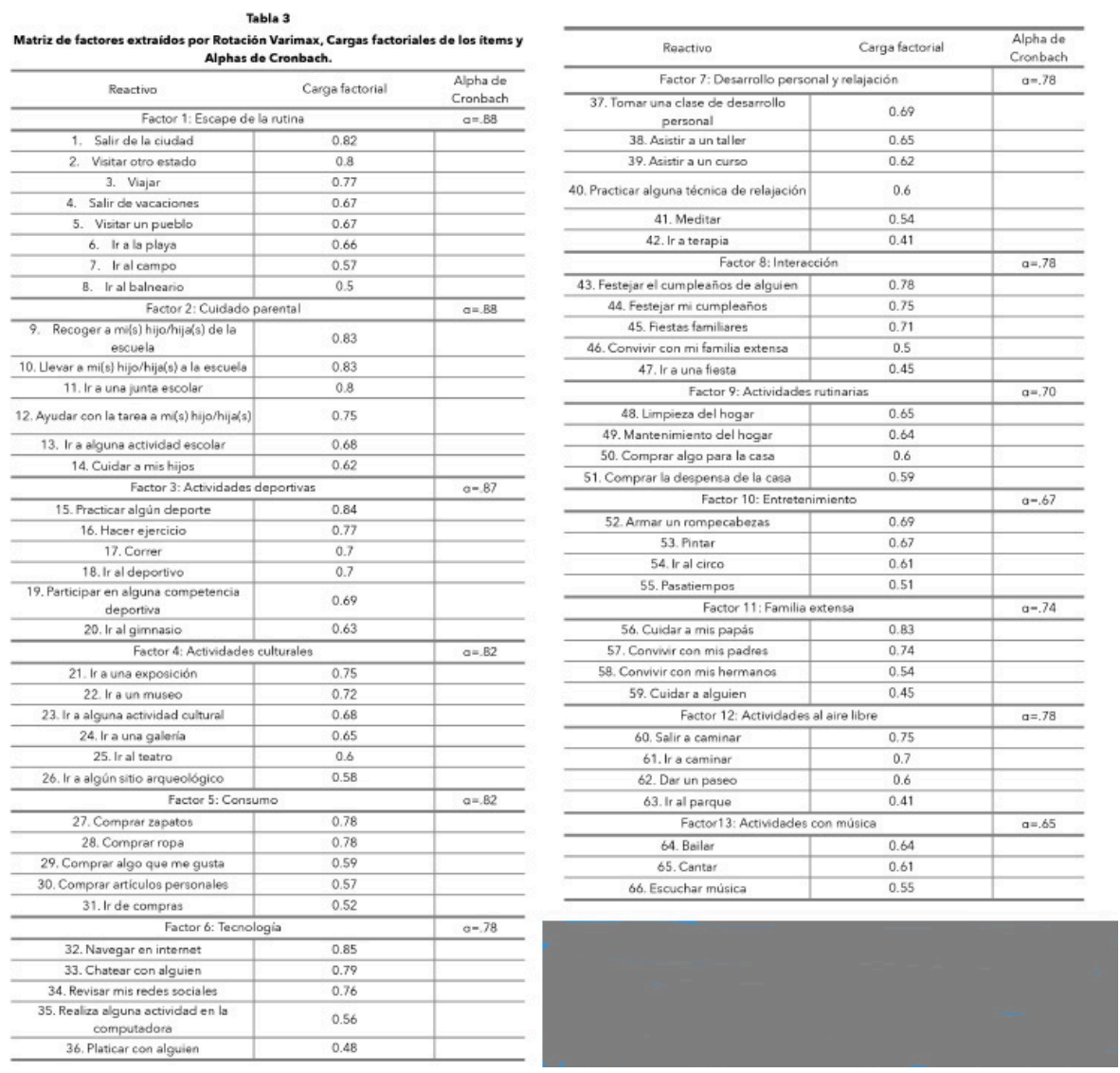

Tabla 3

Matriz de factores extraídos por Rotación Varimax, Cargas factoriales de los ítems y Alpha de Cronbach 
Tabla 4

Coeficientes de Correlación de Pearson entre factores

\begin{tabular}{|c|c|c|c|c|c|c|c|c|c|c|c|c|c|}
\hline & 1 & 2 & 3 & 4 & 5 & 6 & 7 & 8 & 9 & 10 & 11 & 12 & 13 \\
\hline 1. E.R. & - & & & & & & & & & & & & \\
\hline 2. C.P. & $.22^{\star *}$ & - & & & & & & & & & & & \\
\hline 3. A.D. & $.43^{* *}$ & $.25^{* *}$ & - & & & & & & & & & & \\
\hline 4. A.C. & $.54^{\star *}$ & $.25^{\star \star}$ & $.41^{* \star}$ & - & & & & & & & & & \\
\hline 5. C. & $.41^{* *}$ & $.24^{* *}$ & $.34^{* *}$ & $.34^{* *}$ & - & & & & & & & & \\
\hline 6. T. & $.27^{\star \star}$ & $.20^{\star \star}$ & $.23^{\star *}$ & $.23^{* *}$ & $.37^{* *}$ & - & & & & & & & \\
\hline 7. D.P.R. & $.45^{\star *}$ & $.18^{* *}$ & $.45^{* *}$ & $.61^{* \star}$ & $.35^{* *}$ & $.25^{* *}$ & - & & & & & & \\
\hline 8. I. & $.36^{* *}$ & $.27^{* *}$ & $.25^{* *}$ & $.27^{\star * *}$ & $.47^{* *}$ & $.31^{* *}$ & $.19^{* *}$ & - & & & & & \\
\hline 9. A.R. & $.24^{* *}$ & $.39^{* \star}$ & $.27^{\star \star}$ & $.30^{* *}$ & $.49^{* *}$ & $.24^{* *}$ & $.33^{* *}$ & $.28^{* *}$ & - & & & & \\
\hline 10. E. & $.37^{* *}$ & $.30^{* \star}$ & $.38^{* \star}$ & $.46^{* *}$ & $.31^{* *}$ & $.17^{* *}$ & $.35^{* *}$ & $.29^{* *}$ & $.31^{* \star}$ & - & & & \\
\hline 11. F.E. & $.27^{* *}$ & $.43^{* *}$ & $.25^{\star *}$ & $.25^{* \star}$ & $.32^{* *}$ & $.26^{* *}$ & $.19^{* *}$ & $.44^{* *}$ & $.37^{* *}$ & $.34^{* *}$ & - & & \\
\hline 12. A.A.L. & $.44^{\star \star}$ & $.30^{\star *}$ & $.57^{\star \star}$ & $.48^{* \star}$ & $.39^{* *}$ & $.24^{* *}$ & $.43^{* *}$ & $.31^{* *}$ & $.37^{\star \star}$ & $.40^{* *}$ & $.27^{\star \star}$ & - & \\
\hline 13. A.M. & $.24^{* *}$ & $.25^{* *}$ & $.32^{* *}$ & $.30^{* *}$ & $.39^{* *}$ & $.34^{* *}$ & $.30^{* *}$ & $.37^{* *}$ & $.27^{\star \star}$ & $.34^{* *}$ & $.27^{* *}$ & $.35^{* *}$ & - \\
\hline
\end{tabular}

Notas: Los factores corresponden a la Escala de Tiempo Libre; 1. Escape de la rutina, 2. Cuidado parental, 3. Actividades deportivas, 4. Actividades culturales, 5. Consumo, 6. Tecnología, 7. Desarrollo personal y relajación, 8. Interacción, 9. Actividades rutinarias, 10. Entretenimiento, 11. Familia extensa, 12. Actividades al aire libre y 13 . Actividades con música.

${ }^{\star} \mathrm{p}<.05{ }^{\star \star} \mathrm{p}<.001$

Tabla 4

Coeficientes de Correlación de Pearson entre factores

Tabla 5

Diferencias en las actividades de tiempo libre entre padres y madres de familia

\begin{tabular}{|c|c|c|c|c|c|c|c|c|c|}
\hline \multirow{2}{*}{ Actividades de tiempo libre } & \multicolumn{2}{|c|}{ Padres } & \multicolumn{2}{|c|}{ Madres } & & & \multicolumn{2}{|c|}{$95 \%$ IC } & \multirow{2}{*}{$d$ de Coher } \\
\hline & $M$ & $D E$ & $M$ & $D E$ & $t(g \mid)$ & $p$ & 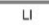 & LS & \\
\hline 1. Escape de la rutina & 2.58 & 0.79 & 2.38 & 0.78 & $3.03(585)$ & 0.01 & 0.06 & 0.32 & 0.25 \\
\hline 2. Cuidado parental & 3.01 & 1.02 & 3.49 & 1.2 & $-5.15(583.28)$ & 0.001 & .0 .65 & .0 .29 & 0.43 \\
\hline 3. Actividades deportivas & 2.38 & 1.06 & 1.94 & 0.86 & $5.45(534.40)$ & 0.001 & 0.28 & 0.59 & 0.45 \\
\hline 4. Actividades culturales & 1.9 & 0.75 & 2.02 & 0.79 & $-1.79(585)$ & 0.07 & -0.24 & 0.01 & 0.15 \\
\hline 5. Consumo & 3.05 & 0.76 & 3.19 & 0.74 & $-2.27(585)$ & 0.05 & .0 .26 & .0 .01 & 0.18 \\
\hline 6. Tecnologia & 3.34 & 1.01 & 3.34 & 0.93 & $.09(585)$ & 0.92 & -0.16 & 0.15 & 0 \\
\hline 7. Desarrollo personal y relajación & 1.82 & 0.71 & 1.94 & 0.85 & $-1.78(581.95)$ & 0.07 & .0 .24 & 0.01 & 0.15 \\
\hline 8. Interacción & 3.5 & 0.84 & 3.61 & 0.77 & $-1.55(585)$ & 0.12 & -0.23 & 0.02 & 0.13 \\
\hline 9. Actividades rutinarias & 3.55 & 0.8 & 4 & 0.67 & $-7.25(545.08)$ & 0.001 & -0.56 & -0.32 & 0.6 \\
\hline 10. Entretenimiento & 1.94 & 0.73 & 1.96 & 0.73 & $-.35(585)$ & 0.72 & -0.13 & 0.09 & 0.02 \\
\hline 11. Fomiliz extensa & 2.74 & 0.97 & 3.18 & 0.98 & $-5.43(585)$ & 0.001 & .0 .59 & -0.28 & 0.45 \\
\hline 12. Actividades al aire libre & 3.09 & 0.95 & 3.01 & 0.93 & $1.02(585)$ & 0.3 & -0.07 & 0.23 & 0.08 \\
\hline 13. Actividades con música & 2.94 & 0.83 & 3.21 & 0.93 & $-3.68(584.95)$ & 0.001 & -0.41 & -0.12 & 0.3 \\
\hline
\end{tabular}

Tabla 5

Diferencias en las actividades de tiempo libre entre padres y madres de familia

Dentro de las actividades a nivel personal, la categoría de Actividades de desarrollo personal y relajación (7) involucra actividades como tomar cursos y talleres, practicar técnicas de meditación y relajación, y acudir a terapia, esta última es la práctica con menor carga factorial, sin embargo, refleja la importancia que ha cobrado en los últimos años. Dichas actividades impactan en la autoestima, la autorrealización y la creatividad (Boltvinik, 2003).

En las actividades de tiempo libre a nivel social, cuya característica es la interacción con el otro, aparecen la categoría Interacción (8), la cual involucra fiestas o reuniones, familiares o en general; y la categoría Familia extensa (11), que implica convivencia y cuidado de la familia, factores que destacan el papel de la familia en la cultura mexicana (Fleiz, Villatoro, Rivera, Medina-Mora, \& Fresán, 1995). Las actividades de tiempo libre favorecen la interacción social (Rodríguez-Suárez \& Agulló- 
Tomás, 2002), sin embargo, las categorías 8 y 11 remiten en su mayoría a la familia extensa, en ambos factores las actividades con menores cargas factoriales son las que remiten a interacción social más allá de la familia: ir a una fiesta y cuidar a alguien.

Los pasatiempos constituyen otra categoría de actividades, el Entretenimiento (10): armar un rompecabezas, pintar e ir al circo; y las Actividades con música (13): cantar, bailar y escuchar música, son actividades que enmarcan el gusto personal, el desarrollo de la creatividad y la formación de la identidad. De igual manera, el Escape de la rutina (1) y las Actividades al aire libre (12), que incluyen viajes y paseos favorecen el descanso, el disfrute y la tranquilidad (Ángel, 2001; Boltvinik, 2003; Damián, 2005; Miquilena, 2011; Munné \& Codina,1996).

Respecto a las Actividades culturales (4), el tiempo libre es una creación cultural, las practicas lúdicas se producen y transforman en el espaciotiempo de cada sociedad en particular, para madres y padres del presente estudio las actividades de disfrute cultural incluyen: exposiciones, museos, galerías, teatros y sitios arqueológicos (Elizalde \& Gomes, 2010).

La práctica de Actividades deportivas (3) tiene efectos a corto y largo plazo, se asocia a bienestar psicológico y emocional y a mejor salud física en general (Cruz-Sánchez et al., 2011).

El Consumo (5) definido como la adquisición de bienes y servicios (Rojek, 2006) ha pasado de ser una función utilitaria a ser una actividad emocionalmente cargada (Cuenca, 2009) que conjunta la identidad personal y social y determina el tipo de actividades de tiempo libre que se realizan y la satisfacción que poseen (Dimanche \& Samdahl, 1994). Para madres y padres de familia el consumo como tiempo libre implica la compra de ropa, zapatos y artículos personales.

La Tecnología (6) ha modificado el estilo de vida, de igual manera ha creado, ampliado y modificado las actividades de tiempo libre (Albrechtsen, 2001). El uso del internet permite tener acceso inmediato a un mundo de posibilidades, mantener contacto con otras personas, revisar las redes sociales y navegar por internet son ejemplos de éstas.

Las correlaciones entre factores muestran asociaciones positivas y significativas. Las correlaciones más altas son tres: la primera entre Actividades culturales (4) y Desarrollo personal y relajación (7) y la segunda entre Actividades culturales (4) y Escape de la rutina (1). Las actividades con contenido cultural son aquellas en las que se visitan lugares con contenido histórico o educativo (sitios arqueológicos, galerías, museos y teatros), estas actividades implican aprendizaje y desarrollo personal, de igual manera el viajar, salir a otro estado o visitar un pueblo (Escape de la rutina) favorece el disfrute de la diversidad de la cultura. La tercera correlación más alta es entre Actividades deportivas (3) y Actividades al aire libre (12), las actividades deportivas a menudo tienen lugar en contacto con la naturaleza, en un bosque o un parque.

Las correlaciones más bajas son dos: entre Tecnología (6) y Entretenimiento (10); y entre Cuidado parental (2) y Desarrollo personal y relajación (7). Aunque no son asociaciones negativas, son débiles. El uso de la tecnología con frecuencia implica actividades pasivas, la persona 
recibe información y no produce en la misma medida, a diferencia del entretenimiento en donde la persona tiene por objetivo la creación (pintar o armar un rompecabezas). Por otra parte, el cuidado parental implica invertir tiempo en los hijos, que implícitamente disminuye la disponibilidad de tiempo para sí mismo (tomar una clase o un taller).

Las tres categorías de actividades que tienen asociaciones más bajas con el resto de actividades son:

Cuidado parental (2), Tecnología (6) e Interacción (8). Dichas categorías de actividades implican el uso del tiempo libre con o en un tercero y pasivamente, diferente a usar el tiempo a libre albedrío, por motivación intrínseca y activamente. Las dos categorías de actividades que tienen asociaciones más altas con el resto de actividades son: Consumo (5) y Entretenimiento (10). La asociación entre el Entretenimiento y una amplia gama de actividades responde a las características de libertad de elección, motivación, diversión y bienestar que comparten. El Consumo por otra parte es una práctica implicada en la vida en general, toda actividad de tiempo libre esta permeada por el consumo: viajar, pertenecer a un deportivo, acudir a un museo, el uso de la tecnología e ir a un concierto implican consumir.

La prueba de diferencias entre hombres y mujeres fue significativa en siete factores; los tamaños de efecto indican que las madres tienen un mayor uso del tiempo libre comparado con los padres en: actividades rutinarias, familia extensa y cuidado parental, comprobando los hallazgos de Mattingly y Sayer (2006) y Offer (2016), es más probable que las madres tengan actividades no remuneradas en el tiempo libre o lo inviertan en el cuidado de los hijos. En lo que respeta a los padres de familia, tienen un mayor uso del tiempo libre en las actividades deportivas, datos que concuerdan con reportados en investigaciones anteriores (Cruz-Sánchez et al., 2011; Martín, Barripedro, Martínez, Jiménez-Beatty, \& Rivero-Herráiz, 2014)

La presente investigación cumple con el objetivo de aportar un instrumento válido y confiable, creado a partir de las actividades de tiempo libre de madres y padres de familia mexicanos. Contar con un instrumento culturalmente relevante es un paso importante para poder evaluar el impacto de esta dimensión (tiempo libre) en múltiples esferas de la realidad social y particularmente en el bienestar. Adicionalmente el trabajo abre futuras líneas de investigación al papel que desempeñan diferentes actividades en la evaluación del tiempo libre en la cultura mexicana. La tecnología como un medio de disfrute, el consumo como una actividad económica que permea el entretenimiento y el disfrute cultural como un medio desarrollo personal.

La investigación presenta una serie de limitaciones, es importante desarrollar investigación en torno al tiempo libre en otras muestras, noviazgo, parejas sin hijos o incorporar la edad de los hijos como una variable importante, la evaluación a través del ciclo de vida (diversas generaciones) y evaluar el tiempo libre en diferentes contextos (rurales y urbanos). 


\section{REFERENCIAS}

Albrechtsen, S. (2011). Technology and lifestyles: Challenges for leisure education in the new millennium. World Leisure Journal, 43(1), 11-19. h ttps://doi.org/10.1080/04419057.2001.9674215

Ángel, M. A. (2001). Del tiempo libre al ocio. Contribuciones desde Coatepec, 1(1), 35-47.

Arteaga, N., Gayet, C., \& Alegría, A. (2016). Uso del tiempo libre, jóvenes y delito en México. Economía, Sociedad y Territorio, XVI(52), 623-650.

Boltvinik, J. (2003). Conceptos y medición de la pobreza. La necesidad de ampliar la mirada. Papeles de Población, 9(38), 9-25.

Claxton, A., \& Perry-Jenkins, M. (2008). No fun anymore: Leisure and marital quality across the transition to parenthood. Journal of Marriage and Family, 70(1), 28-43. https://www.doi.org/10.1111/j.1741-3737.2007.0 0459.x

Cruz-Sánchez, E., Moreno-Contreras, M. I., Pino-Ortega, J., \& MartínezSantos, R. (2011). Actividad física durante el tiempo libre y su relación con algunos indicadores de salud mental en España. Salud Mental, 34(1), 45-52.

Cuenca, J. (2009). Tiempo ejemplar: El ocio y los mundos vivenciales. En M. Cuenca \& E. Aguilar (Eds.), El tiempo de ocio: transformaciones y riesgos en la sociedad apresurada (pp. 57-73). Bilbao, España: Universidad de Deusto.

Damián, A. (2004). La pobreza de tiempo: Conceptos y métodos para su medición. En J. Boltvinik \& A. Damián (Eds.), La pobreza en México y el mundo. Relidades y desafios (pp. 481-518). México: Siglo XXI.

Damián, A. (2005). El costo de ser pobre de tiempo. Economia Informa, 48-60.

Dimanche, F., \& Samdahl, D. (1994). Leisure as a symbolic consumption: A conceptualization and prospectus for future research. Leisure Sciences, 16(2), 119-129. https://www.doi.org/10.1080/01490409409513224

Elizalde, R., \& Gomes, C. (2010). Ocio y recreación en América Latina: Conceptos, abordajes y posibilidades de resignificación. Polis, Revista de la Universidad Bolivariana, 9(26), 1-15.

Fleiz, C., Villatoro, J. A., Rivera, E., Medina-Mora, M. E., \& Fresán, A. (1995). La interacción padres-hijos: Una evaluación de la percepción de los padres. Revista de Psicología Social y Personalidad, XI(1), 31-40.

García-Méndez, M., Rivera-Aragón, S., Díaz-Loving, R., \& Reyes Lagunes, I. (2015). Continuidad y cambio en la familia: Factores intervinientes. México: Manual Moderno.

Gutiérrez, E. (2008). Tiempo libre y calidad de vida desde el sí mismo docente. Revista Mexicana de Investigación Educativa, 13(38), 867-890.

Hernández, R., Fernández, C., \& Baptista, P. (2010). Metodología de la investigación. México: McGraw Hill.

Instituto Nacional de Estadística y Geografía [inegi] (2014). Encuesta Nacional sobre Uso del Tiempo (enut). Recuperado de www.beta.inegi.org.mx/ proyectos/enchogares/especiales/enut/2014/

Lee, Y. G., \& Bhargava, V. (2004). Leisure time: Do married and single individuals spend it differently? Family and Consumer Sciences Research Journal, 32(3), 254-274. https://doi.org/10.1177/1077727X03261631 
Martín, M., Barripedro, M. I., Martínez, J., Jiménez-Beatty, J. E., \& RiveroHerráiz, A. (2014). Diferencias de género en los hábitos de actividad física de la población adulta en la comunidad de Madrid. Revista Internacional de Ciencias del Deporte, $X(38), 319-335$.

Mattingly, M. J., \& Sayer, L. C. (2006). Under pressure: Gender differences in the relationship between free time and feeling rushed. Journal of Marriage and Family, 68(1), 205-221. https://doi.org/10.1111/j.1741-3737.2006 $.00242 . \mathrm{x}$

Miquilena, D. (2011). Recreación laboral: Su efecto motivacional en los trabajadores. Observatorio Laboral Revista Venezolana, 4(8), 37-51.

Munné, F., \& Codina, N. (1996). Psicología social del ocio y el tiempo libre. En J. L. Álvaro, A. Garrido \& J. R. Torregrosa (Eds.), Psicología social aplicada (pp. 429-448). Madrid, España: McGraw Hill.

Offer, S. (2016). Free time and emotional well-being: Do dual-earner mothers and fathers differ? Gender \& Society, 30(2), 213-239. https://doi.org/10. $1177 / 0891243215596422$

Reyes Lagunes, L. I., \& García y Barragan, L. F. (2008). Procedimiento de validación psicométrica culturalmente relevante. En S. Rivera-Aragón, R. Díaz- Loving, R. Sánchez-Aragón \& L. I. Reyes Lagunes (Eds.), La psicología social en México, XII (pp. 625- 636). México: Amepso.

Rodríguez-Suárez, J., \& Agulló-Tomás, E. (2002). Psicología social y ocio: Una articulación necesaria. Psicothema, 14(1), 124-133.

Rojek, C. (2006). Leisure and consumption. Leisure/ Loisir, 30(2), 475-486. ht tps://doi.org/10.1080/14927713.2006.9651368

Romo, C. (2003). Tiempo de libertad para la libertad. Algunos apuntes sobre la crítica a la alienación del ocio en la sociedad industrial avanzada. Baetica. Estudios de Historia Moderna y Contemporánea, 773-790.

Shaw, S. M. (2008). Family leisure and changing ideologies of parenthood. Sociology Compass, 2(2), 688-703. https://doi.org/10.1111/j.1751-9020. 2007.00076.x

Shaw, S. M., \& Dawson, D. (2001). Purposive leisure: Examining parental discourses on family activities. Leisure Sciences: An Interdisciplinary Journal, 23(4), 217-231. https://doi.org/10.1080/01490400152809098

Zabriskie, R. B., \& McCormick, B. P. (2001). The influences of family leisure patterns on perceptions of family functioning. Family Relations, 50(3), 281-289. https://doi.org/10.1111/j.1741-3729.2001.00281.x 